\title{
Estrategias utilizadas por un grupo de mujeres mexicanas para cuidar su salud emocional: autoatención y apoyo social
}

\author{
Shoshana Berenzon-Gorn, Dra Psic,(I) Nayelhi Saavedra-Solano, Dra Antr Soc, (I) \\ Sarahí Alanís-Navarro, M en Psic.(I)
}

\begin{abstract}
Berenzon-Gorn S, Saavedra-Solano N, Alanís-Navarro S. Estrategias utilizadas por un grupo de mujeres mexicanas para cuidar su salud emocional: autoatención y apoyo social.
\end{abstract} Salud Publica Mex 2009;5 I:474-48I.

\section{Resumen}

Objetivo. Conocer las prácticas de autocuidado/autoatención utilizadas por un grupo de mujeres para aliviar malestares emocionales. Material y métodos. Participaron 23 mujeres, habitantes de la Ciudad de México. Se realizaron entrevistas focalizadas mediante la técnica de entrevista de investigación social, cuyo propósito es favorecer la producción del discurso continuo sobre un tema determinado. La información se analizó con la técnica de categorización de significados, que consiste en clasificar el contenido de cada entrevista en categorías mutuamente excluyentes. Resultados. Las prácticas de autoatención reúnen: conductas de autocontrol, remedios caseros, automedicación y actividades de relajación. El apoyo social proviene principalmente de otras mujeres; la pareja no se considera un apoyo significativo. Conclusiones. Las mujeres encuentran estrategias que, si bien no solucionan por completo sus malestares emocionales, les permiten sobrellevar las situaciones vinculadas con éstos.

Palabras clave: autocuidado; apoyo social; mujeres; salud mental; México
Berenzon-Gorn S, Saavedra-Solano N,Alanís-Navarro S. Strategies for taking care of emotional health among the Mexican women population: self-care and social support. Salud Publica Mex 2009;5 I:474-48 I.

\section{Abstract}

Objective. To describe what kind of self care practices a group of women use to alleviate their emotional disorders. Material and Methods. A group of 23 women living in Mexico City answered open-ended questions about self care strategies. Focused interviews were carried out using a social research interview technique designed to encourage continuous discourse on a set topic. Data was analyzed using a significant classification technique, which involves placing the contents of each interview in mutually exclusive categories. Results. Self care practices reported included self-control, home remedies, self-medication, relaxing activities and social support. Social support is mainly obtained from other women, since partners were not regarded as providing meaningful support. Conclusions. Self care practices start once the malaise begins and stop when the malaise ends.Although the strategies women use do not entirely solve their emotional disorders, they enable them to cope with such situations.

Key words: self-care; social support; women; mental health; Mexico

(I) Instituto Nacional de Psiquiatría Ramón de la Fuente. México, DF, México.

Fecha de recibido: 8 de octubre de 2008 - Fecha de aceptado: 23 de julio de 2009 Solicitud de sobretiros: Dra. Shoshana Berenzon Gorn. Instituto Nacional de Psiquiatría. Calz. México Xochimilco I0I, col. San Lorenzo Huipulco.Tlalpan I4370 México, DF, México. Correo electrónico berenz@imp.edu.mx. 
$E^{n}$ las sociedades actuales coexisten distintos saberes médicos y, en consecuencia, son posibles diversos sistemas de atención. Un rasgo inherente a estos sistemas es que las personas utilizan de manera simultánea o intermitente distintas estrategias de cuidado para tratar un malestar.

Se han propuesto varios modelos teóricos para comprender las diferentes formas en que se combinan y relacionan las prácticas curativas y los servicios formales e informales de salud.

Uno de los modelos más completos es el que propuso Haro ${ }^{1}$ mediante el cual se describe el sistema plural de atención que distingue cuatro estructuras básicas: a) autocuidado y autoatención, b) autoayuda y autogestión, c) atención alternativa y d) atención médica profesional. Estas cuatro formas se conjugan, complementan y yuxtaponen y crean un espacio en el cual las personas definen las enfermedades y seleccionan tanto a los sanadores como a las alternativas curativas.

En el modelo se menciona que el autocuidado y la autoatención son elementos centrales para el funcionamiento de las demás formas de atención a la salud; su relevancia radica en que estas prácticas constituyen una actividad constante, aunque intermitente. ${ }^{2}$

El concepto de autocuidado se ha analizado desde diferentes disciplinas. La psicología lo ha vinculado con factores internos de salud e integrado a nociones como autoconcepto y las creencias sobre la salud. Los estudios provenientes de la biomedicina suelen asociar el autocuidado con la atención y tratamiento de la enfermedad y se han enfocado en la transferencia de la responsabilidad de algunos cuidados médicos hacia los pacientes. El campo de la salud pública se ha ocupado de medir factores de riesgo y asociarlos con conductas identificables de autocuidado que deben modificarse, evitarse o promoverse. Otras disciplinas interesadas en el estudio del autocuidado son la sociología y la enfermería. ${ }^{3}$

Si bien estos enfoques poseen diferencias, coinciden en señalar que el autocuidado se conforma con prácticas realizadas principalmente de manera individual, centradas en aspectos preventivos o de promoción de la salud y efectuadas como parte de las actividades cotidianas.

Otra disciplina interesada en el autocuidado es la antropología médica, pero se ha distinguido de los enfoques antes mencionados porque algunos de sus exponentes han cuestionado la visión individualista de este concepto. ${ }^{1,2}$ Es por ello que proponen el concepto de autoatención, en el cual se incluyen las prácticas de autocuidado y lo definen como "las representaciones y prácticas que la población utiliza a nivel de sujeto y grupo social para diagnosticar, explicar, atender, controlar, aliviar, aguantar, curar, solucionar o prevenir los procesos que afectan su salud en términos reales o imaginarios, sin la intervención central, directa e intencional de curadores profesionales, aun cuando éstos pueden ser la referencia de la actividad de autoatención. Incluye los hábitos personales, la actividad física y la automedicación de fármacos de patente y de 'remedios caseros' ". ${ }^{2}$ Dentro de este constructo también se engloban algunas redes sociales de apoyo como la familia, los amigos y los vecinos y, en consecuencia, sobresale el carácter social de este tipo de prácticas. ${ }^{1} \mathrm{El}$ análisis del material que más adelante se presenta se basa en esta definición.

Las prácticas de autoatención se encuentran en estrecha relación con el ámbito doméstico, en el cual las figuras destacadas en la reproducción de tales prácticas son las mujeres en su papel de madres y esposas. Ellas se encargan de diagnosticar el padecimiento, establecer su gravedad o levedad, aplicar los primeros tratamientos, utilizar yerbas, infusiones o fármacos para el cuidado de la familia y para ellas mismas. La figura femenina también suele presentarse como principal fuente de decisiones para elegir entre una terapéutica y otra. ${ }^{4-5}$

Diversos estudios señalan que las prácticas de autoatención se usan con frecuencia para atender malestares, relacionados sobre todo con trastornos depresivos y de ansiedad y los reporta con mayor frecuencia la población femenina. ${ }^{6}$ Las prácticas de autoatención que más usan las mujeres son las actividades físicas, la automedicación y el uso de remedios caseros. ${ }^{78}$

Las encuestas realizadas a escala nacional sobre salud mental han mostrado que las mujeres prefieren acudir en primera instancia a un amigo o un familiar y en segundo lugar a los remedios caseros o la automedicación. ${ }^{910}$ Otras estrategias empleadas a menudo por la población femenina son "tratar de descansar", "tomar el sol un rato", "hacer ejercicio" o "controlar su nervios" para sentirse mejor. ${ }^{11}$

Las investigaciones mencionadas estuvieron encaminadas, de manera preferente, hacia el análisis de trastornos mentales diagnosticados. Para este trabajo se ha optado por utilizar el término de malestar emocional para comprender los problemas de salud de las mujeres, puesto que guarda mayor afinidad con la perspectiva social del término autoatención. Para Burin,, ${ }^{12}$ el malestar emocional en las mujeres se relaciona con el modo en que perciben sus padecimientos, de tal forma que no les permite sentirse saludables pero tampoco enfermas, una percepción ambigua de incomodidad y desasosiego.

Las características y el desarrollo de este tipo de malestares no siempre coinciden con entidades determinadas por la biomedicina o la psicología porque se construyen a partir de los conocimientos legos. Además, con el concepto de malestar emocional se reconoce la dificultad para fragmentar en unidades discretas el 
cúmulo de vivencias cotidianas (relaciones hostiles, precariedad económica, contradicciones morales, etc.) que actúan sobre tales experiencias. ${ }^{13}$

Con base en las anteriores consideraciones, el objetivo de este trabajo fue conocer las prácticas de autoatención utilizadas por un grupo de mujeres de la Ciudad de México para aliviar malestares emocionales.

\section{Material y métodos}

\section{Población}

En el estudio participaron 23 mujeres habitantes de seis colonias de la Ciudad de México que informaron utilizar uno o varios recursos para atender un malestar emocional. La selección de las informantes se realizó a través del muestreo teórico, ${ }^{14}$ en el cual el número de personas entrevistadas no es lo más importante, sino la información que aporten para interpretar el tema de interés.

\section{Procedimiento}

Se realizaron entrevistas focalizadas mediante la técnica de entrevista de investigación social, la cual tiene como propósito favorecer la producción del discurso continuo y posee una cierta línea argumental sobre un tema determinado en el marco de la investigación. ${ }^{15}$

Para recopilar la información se elaboró una guía de entrevista, ${ }^{*}$ que incluyó los siguientes temas clave: a) historial clínico del padecimiento; b) carrera o trayectoria de búsqueda de atención, c) características de los recursos o tratamientos utilizados; d) percepción social y cultural de la salud mental; e) percepción hacia el profesional y el servicio otorgado (relación médicopaciente); y f) eficacia terapéutica. Sin embargo, para cubrir los objetivos de este escrito sólo se analizaron los principales motivos y características del uso de remedios caseros, vitaminas, medicinas, etc., así como la búsqueda de ayuda con familiares, amigos $u$ otros miembros de la red de apoyo.

Todas las entrevistas se llevaron a cabo en las viviendas de las participantes; la mayor parte de éstas se efectuó en una sesión y duró alrededor de 90 minutos.

\footnotetext{
* La guía de entrevista es una lista de tópicos y áreas generales necesarias para la interacción verbal que el entrevistador sistematiza con dos cometidos principales: reflexionar sobre la prosodia del habla que manifiesta el entrevistado y, al mismo tiempo, organizar los temas sobre los que se formulan preguntas en la entrevista; es en particular útil para que al inicio de la conversación el entrevistado se ajuste al plan temático. ${ }^{16}$
}

En algunos casos fue necesario más de un encuentro para agotar los diversos temas. Durante el primer contacto, se hizo una presentación en la cual se explicaron los objetivos de la investigación, motivos de la entrevista y el carácter confidencial de la información, y se solicitó autorización para grabar las conversaciones. El trabajo de campo comprendió el periodo de agosto de 2006 a diciembre de 2007.

\section{Análisis de la información}

Se elaboró una serie de categorías para clasificar la información de los temas tratados en las conversaciones; esta agrupación se basó en la técnica de "categorización de significados" que propuso Kvale. ${ }^{17}$ Por medio de esta técnica, cada entrevista se codifica en una serie de categorías mutuamente excluyentes, lo que permite estructurar las narraciones en unidades de información que facilitan la comprensión del tema de interés, así como su ocurrencia a lo largo del discurso. Dos personas codificaron las entrevistas por separado para luego comparar las categorizaciones obtenidas. Las discrepancias en la codificación se resolvieron con la discusión y mediante una revisión conjunta de los relatos originales.

Después de este proceso se contó con un total de 116 categorías; en este análisis se consideraron las siguientes:

1. Fuerza de voluntad: hace referencia a las diversas acciones de autocontrol empleadas para disminuir o eliminar el malestar provocado por algún síntoma, así como "aguantarse" y esperar hasta que el problema desaparezca por sí solo. ${ }^{18}$

2. Remedios caseros: entre los más comunes se encuentran los baños, infusiones y pomadas; por lo general se preparan con hierbas, flores y plantas. Casi siempre los recomiendan familiares o amigos cercanos.

3. Automedicación: es el consumo de fármacos sin prescripción médica e incluye las vitaminas, complementos alimenticios y diversos fármacos. ${ }^{19}$

4. Ejercicios y otras prácticas: incluyen actividades físicas, de relajación y deportes; algunos ejemplos son ejercicios aeróbicos, yoga, caminata y ejercicios de relajación, entre otros. Para las categorías antes mencionadas se recopiló información sobre el tipo de remedios, medicamentos y prácticas utilizadas, la fuente de información, conocimiento de los efectos (positivos y negativos) y la percepción sobre los beneficios del uso.

5. Redes informales de apoyo: aluden a las relaciones personales basadas en vínculos afectivos que no se conformaron para brindar atención sanitaria. 
Comprenden a la familia extensa, las amistades, compadrazgos, compañeros de trabajo o estudio y grupos vecinales. ${ }^{1}$ Para esta categoría se recopiló información sobre las personas a quienes se recurre, tipo de ayuda solicitada o recibida y grado de satisfacción por la ayuda recibida. Para analizar la información sobre las redes informales de apoyo se retomó la propuesta de Broadhed, ${ }^{20}$ quien plantea tres dimensiones del apoyo otorgado: el "apoyo afectivo o emocional", que se puede concretar en expresiones de amor, cariño, estima, empatía, etc., el "apoyo material o instrumental", como las acciones o materiales proporcionados por otras personas que permiten cumplir las responsabilidades cotidianas o que resuelven problemas prácticos (prestar dinero, efectuar tareas domésticas, cuidar a los hijos, etc.), y "el apoyo informativo" a través del cual las personas reciben información consejo o guía. Estos tres tipos de apoyo se encuentran altamente vinculados y en la mayoría de los casos se proporcionan de manera simultánea.

\section{Consideraciones éticas}

El Comité de Ética en Investigación del Instituto Nacional de Psiquiatría Ramón de la Fuente aprobó el proyecto de investigación. De acuerdo con las normas estipuladas por este comité, se consideró que el proyecto no representaba riesgos mayores y por lo tanto se podía obtener el consentimiento informado de manera verbal. Para solicitar el consentimiento informado de los participantes, se les mencionaron los objetivos y motivos de la investigación, el carácter confidencial de los datos, y también se les pidió autorización para grabar las entrevistas y divulgar la información recolectada.

\section{Resultados}

Como ya se ha mencionado, se entrevistó a 23 mujeres; la edad promedio fue de 39 años. Sin embargo, seis de las entrevistadas tenían 50 años o más. Respecto del nivel de estudios, ocho mujeres alcanzaron estudios de primaria, siete de secundaria o preparatoria y seis tenían estudios de licenciatura y posgrado. Más de la mitad de las integrantes del grupo mencionó tener como actividad principal las labores del hogar; ocho trabajaban como empleadas de una oficina gubernamental y una de ellas contaba con un negocio informal propio (cuadro I).

\section{Malestares emocionales}

Los malestares emocionales que refirieron las mujeres fueron cansancio permanente, intranquilidad, ner-

Cuadro I

Características sociodemográficas de las entrevistadas

\begin{tabular}{|c|c|c|c|c|c|c|c|}
\hline $\begin{array}{c}\text { Número } \\
\text { de entrevista }\end{array}$ & Seudónimo & Edad & Ocupación & Escolaridad & Estado civil & Religión & $\begin{array}{c}\text { Tipo de seguro } \\
\text { médico }\end{array}$ \\
\hline I & Mireya & 54 & ama de casa & $6^{\circ}$ de primaria & divorciada & testigo de Jehová & IMSS \\
\hline 2 & Mariana & 67 & ama de casa & licenciatura & viuda & católica & IMSS \\
\hline 3 & Amanda & 49 & ama de casa & $3^{\circ}$ de preparatoria & casada & católica & IMSS \\
\hline 4 & Gabriela & 36 & empleada & licenciatura & soltera & católica & ninguno \\
\hline 5 & Greta & 43 & empleada & posgrado & casada & otra & IMSS \\
\hline 6 & Dulce & 22 & empleada & $1^{\circ}$ de preparatoria & casada & católica & ninguno \\
\hline 7 & Miriam & 33 & $\begin{array}{l}\text { trabaja por cuenta } \\
\text { propia }\end{array}$ & $3^{\circ}$ de preparatoria & casada & católica & IMSS \\
\hline 8 & Alejandra & 46 & empleada & rehúsa & viuda & católica & ninguno \\
\hline 9 & Lourdes & 55 & ama de casa & $3^{\circ}$ de secundaria & viuda & católica & IMSS \\
\hline 10 & Liliana & 42 & ama de casa & $3^{\circ}$ de secundaria & casada & católica & IMSS \\
\hline 11 & Blanca & 58 & ama de casa & $3^{\circ}$ de preparatoria & separada & católica & ninguno \\
\hline 12 & Rebeca & 46 & empleada & licenciatura & divorciada & católica & ninguno \\
\hline 13 & Griselda & 38 & ama de casa & $\mathrm{I}^{\circ}$ de primaria & unión libre & católica & ninguno \\
\hline 14 & María & 60 & ama de casa & $\mathrm{I}^{\circ}$ de primaria & divorciada & católica & ninguno \\
\hline 15 & Amalia & 38 & empleada & $3^{\circ}$ de secundaria & separada & católica & IMSS \\
\hline 16 & Mercedes & 39 & ama de casa & $6^{\circ}$ de primaria & casada & católica & ninguno \\
\hline 17 & Lupe & 39 & empleada & posgrado & soltera & ninguna & ISSSTE \\
\hline 19 & Rocío & 37 & empleada & licenciatura & soltera & ninguna & ninguno \\
\hline 20 & Elizabeth & 35 & ama de casa & $6^{\circ}$ de primaria & casada & católica & ninguno \\
\hline 21 & Carmen & 37 & ama de casa & $3^{\circ}$ de primaria & unión libre & $\begin{array}{l}\text { creyente } \\
\text { en Jesús }\end{array}$ & ninguno \\
\hline 22 & Cristina & 62 & ama de casa & $6^{\circ}$ de primaria & viuda & católica & IMSS \\
\hline 23 & Bertha & 29 & ama de casa & $6^{\circ}$ de primaria & casada & católica & ninguno \\
\hline
\end{tabular}


viosismo, conflictos con los integrantes de la familia (sobre todo la pareja y los hijos), pérdida de fuerza para realizar sus actividades y estrés. Aunado a lo anterior, las entrevistadas señalaron sentirse afectadas por preocupaciones económicas y la inseguridad y la violencia percibidas en la Ciudad de México.

\section{Las prácticas de autoatención}

\section{Fuerza de voluntad}

Entre las primeras acciones reportadas por las entrevistadas para tolerar los síntomas del malestar se encuentran las "conductas de autocontrol" que se relacionan con las distintas maneras de utilizar la fuerza de voluntad para atenuar el malestar. En las frases "yo puedo sola", "no es nada grave" y "con el tiempo se va a quitar" se refleja que los problemas de salud y enfermedad quedan integrados en la vida cotidiana como un suceso más sobre el que debe tenerse control para no generar mayores conflictos.

Entonces, yo siempre estaba cansada, en esa época siempre estaba cansada, por eso ¿no? Yo creo que porque hacía un, un muy fuerte esfuerzo de auto control para no, este, pues que no se fuera todo al demonio ¿no?, entonces si era como indispensable que no, o sea, no dejarse caer. (Entrevista 5)

\section{Uso de remedios caseros}

Entre los principales motivos para recurrir a los remedios caseros se mencionaron los siguientes: tranquilizarse, controlar los nervios, conciliar el sueño y ayudar a relajarse. También se utilizaron estos recursos porque percibían que al ser naturales no hacían daño o bien por "costumbre". Resulta pertinente señalar que en la mayor parte de los casos, el uso de remedios caseros se presentó de manera conjunta con la práctica de otras acciones de autoatención y otros recursos terapéuticos.

Las entrevistadas mencionaron que suelen utilizar plantas con propiedades tranquilizantes o relajantes, como azahar, tila, valeriana, toronjil, flor de manita, pasiflora, "té de doce flores", lechuga y rosas (cuadro II). Casi todas estas plantas se preparan como infusiones o tés; sólo en el caso de la lechuga y las rosas señalaron usarlas en forma de baños. Por lo general, el conocimiento de este tipo de remedios proviene del seno familiar o personas cercanas, como amigos y compañeros de trabajo.

[Las usé] Porque yo ya tenía problemas con mi esposo, ya nos estábamos separando, entonces estaba yo muy estresada, muy. No sé (...) Me lo recomendó una amiga [el baño de rosas], que siempre he platicado con ella y que sabe de mi problema (...) Me dijo que comprara los pétalos de la rosita y ponerlos a hervir con agua y tenerlo listo y ya cuando se acaba de hacer su baño normal, ya como último se echa uno esa agua de rosas. (Entrevista 15)

\section{Automedicación}

Los productos que emplearon con mayor frecuencia, sin la intervención de un especialista en salud, fueron vitaminas, complementos alimenticios y medicamentos naturales o químicos que incluían desde analgésicos hasta psicofármacos. Las personas usaron los fármacos de fácil acceso; los que se hallaban en casa (casi siempre porque en algún momento se prescribieron como parte de un tratamiento específico) y los productos que promocionan los medios de comunicación. Fue común que los familiares, amigos y encargados de las farmacias recomendaran los productos.

Usaron estos productos cuando consideraron que la dolencia o el síntoma no requerían atención médica o para prevenir un malestar recurrente. Otras razones fueron "mejorar el rendimiento", "disminuir el cansancio", "relajarse", "fortalecer el cuerpo" y revertir un déficit de vitaminas. También los utilizaron para controlar "el estrés", "la tristeza", "la ansiedad" o "el insomnio"; las entrevistadas indicaron que recurrieron a ciertos complementos y medicamentos porque son naturales $y$, en general, percibidos como eficaces.

\section{Cuadro II \\ Nombre CIENTífICO DE LAS PLANTAS UTILIZADAS POR LAS MUJERES ENTREVISTADAS}

\begin{tabular}{ll} 
Nombre común & \multicolumn{1}{c}{ Nombre científico } \\
\hline Azahar & Familia Rutaceae. Citrus aurantium L. \\
\hline Flor de tila & Familia Tiliaceae. Tilia mexicana Schlechtendal \\
\hline Valeriana & $\begin{array}{l}\text { Familia Valerianaceae. Valeriana edulis Nutt. subsp. procera } \\
\text { (Kunth)F.G.Mey. }\end{array}$ \\
\hline Toronjil & $\begin{array}{l}\text { Familia Labiate. Agastache mexicana (Kunth) Lint y } \\
\text { Epling }\end{array}$ \\
\hline Flor de manita & $\begin{array}{l}\text { Familia Sterculiaceae. Chiranthodendron pentadactylon } \\
\text { Lam. }\end{array}$ \\
\hline Pasiflora & Familia Passifloraceae. Passiflora suberosa L. \\
\hline Lechuga & Familia Compositae. Lactuca sativa L \\
\hline Rosa & Familia Rosaceas. Aff. Rubiginosa L.
\end{tabular}

Fuente de información consultada: Referencia 21 
Hasta ahorita todavía si yo la pido, me lo dan, aunque ya no esté en tratamiento con la doctora, le decía yo ya tengo como un año con una cajita de imipramina y sólo cuando me altero mucho me tomo una, una pastillita y ya. (Entrevista 1)

Las mujeres reportaron diversas percepciones acerca de los beneficios que han obtenido después de usar remedios caseros o fármacos, desde una mejoría considerable hasta la ausencia de cambios notorios. En términos generales, las entrevistadas lograron relajarse; sin embargo, aseguraron que ese cambio de estado no resolvió por completo el problema. Para algunas mujeres, el uso contribuyó para que se sintieran mejor o bien.

\section{Ejercicios y otras prácticas}

Se distinguieron tres grupos de prácticas; el primero incluye la gimnasia, danza, natación, aeróbicos, spinning, caminata al aire libre, entre otras actividades de índole deportiva. Las entrevistadas recurrieron a un "saber generalizado" acerca de los beneficios asociados con las prácticas físicas. Este conocimiento se muestra en frases como "yo creo que todo mundo lo sabe, que es beneficioso el caminar...".

En el segundo grupo se encuentra escuchar música, tejer, leer, escribir, además de otras actividades recreativas, como elaborar collares u objetos decorativos ("manualidades"). Se incluyeron las labores domésticas porque algunas mujeres mencionaron que son una forma de sobrellevar sus problemáticas emocionales. ..." [al] lavar y lavar, en el agua se van mis broncas".

El tercer grupo está conformado por la yoga, tai-chi y diversas formas de meditación basadas en el control de la respiración y la imaginería. Estas prácticas tienen en común que las mujeres dedican entre 15 y 45 minutos a cerrar los ojos y concentrarse en su inhalación y exhalación. Las entrevistadas se iniciaron en estas actividades mediante sugerencias de amigos y familiares o por prescripción médica.

Entre los beneficios que señalaron, al margen del grupo de actividades, se identificaron los siguientes: distraerse, relajarse o enfrentar el estrés, contar con un espacio personal, sentirse ágiles, despejarse de los problemas cotidianos y canalizar las emociones como ira o tristeza.

...[hacer yoga y caminar] me hacían bien, me ayudaban mucho a relajarme, para desestresarme, para tener otros caminos, otras actividades, no sólo las actividades de la casa, de la familia, del quehacer cotidiano que es estresante y agobiante. (Entrevista 2)

\section{Las redes de apoyo: familia y amigos}

Como ya se mencionó, se analizaron tres dimensiones del apoyo otorgado: "apoyo afectivo o emocional", "apoyo material o instrumental" y "apoyo informativo".

Un claro ejemplo del apoyo "afectivo o emocional" se observa cuando algún miembro de la red hace comentarios como "échale ganas", "no te preocupes, todo está en ti" o "cambia esa cara". Estos comentarios forman parte de la creencia cultural en la cual los padecimientos emocionales se perciben como una debilidad de carácter que puede solucionarse mediante el autocontrol.

Sí, que le echara muchas ganas, que yo le echara muchas ganas porque yo era la que estaba malita y que no me preocupara y que, o sea, que buscara que yo me sintiera bien porque ellas [madre y amigas] no me podían ayudar así porque el mal yo lo traía dentro de mí. (Entrevista 21)

Las entrevistadas también reconocen el apoyo afectivo en las manifestaciones de amor o empatía y en las respuestas recibidas al buscar atención, compresión, desahogo, soporte, catarsis e identificación.

El apoyo "material o instrumental" reportado con mayor frecuencia fue recibir ayuda para solventar problemas económicos. Las entrevistadas también informaron que los familiares apoyaron en el cuidado de los hijos y la realización de labores domésticas, como preparar la comida o limpiar la casa.

En relación con el "apoyo informativo" recibido, las entrevistadas destacaron los consejos y recomendaciones. Fue recurrente que los consejos llevaran a la búsqueda de ayuda con un especialista, como psicólogo o médico tradicional, o bien que acudieran a la práctica de alguna actividad como el yoga.

Me decía mi mamá que yo era medio loca, medio estresada y medio enojona y a lo mejor mi mamá me decía pues ve a tal parte, a mí esto o el otro. Pues yo ahora después de casada yo continué con esas enseñanzas... (Entrevista 2)

Cualesquiera que sean la dimensión o el tipo de ayuda recibidos, las mujeres comentaron que las amigas, la madre y los hermanos han sido su principal fuente de apoyo. Resulta pertinente mencionar que, aun cuando casi todas ellas están casadas o viven con alguien, en muy pocos casos la pareja se percibió como una fuente de ayuda en caso de malestar emocional; la figura del padre tampoco se percibió como un buen punto de apoyo.

Los relatos de las mujeres confirman que las redes informales de apoyo juegan un papel importante en la 
construcción y validación de la gravedad del malestar. Las entrevistadas señalaron que fueron sus amigos o familiares los primeros en notar su "mal semblante", "rarezas" o "cambios en la apariencia" y, debido a ello, se las inquirió por la posibilidad de estar enfermas. La información recopilada también demuestra que las redes de apoyo cumplen funciones cuidadoras tan importantes como las otorgadas por cualquier sistema de atención y, en muchas ocasiones, son las únicas que proporcionan los cuidados necesarios.

Yo nunca me di cuenta y si mi mamá no hubiera insistido en que yo tenía una conducta rara, obviamente yo estaba viviendo otra realidad, y para mí yo estaba normal y yo veía raros a mis papás ¿por qué están insistiendo en que yo estoy rara?, cuando para mí todo era normal, si no tengo nada y ellos empezaron a ver esta conducta rara y me decían oye vamos al médico que mira y yo les decía ¿para qué vamos a ir al médico?... (Entrevista 4)

\section{Discusión}

La forma como las mujeres llevaron a la práctica las diversas estrategias de autoatención muestra que estos recursos suelen estar presentes desde la aparición del malestar emocional hasta su resolución final .

Los relatos presentados apoyan la propuesta de Hernández, ${ }^{22}$ según la cual las mujeres se apropian la información que reciben de sus madres, abuelas y otras mujeres, o bien la que procede de otras fuentes, como los servicios de salud, las medicinas alternativas, los encargados de las farmacias o, incluso, la que generan los medios de comunicación. Ellas reinterpretan la información, construyen con ella un acervo de conocimientos que utilizan para explicar las causas del malestar y le dan un significado que les permite integrarlo a su historia, adoptar una postura frente al trastorno y decidir la forma de atenderlo o combatirlo. En las estrategias de autoatención, dicho acervo entra en función en su totalidad.

Este trabajo permitió analizar aspectos que no se habían considerado al principio de la investigación: actividades y actitudes que se encuentran incorporadas en la vida diaria, como lavar, asear la casa y la fuerza de voluntad. Salgado de Snyder ${ }^{18}$ plantea que si bien este tipo de acciones no soluciona el padecimiento, sí les permite a muchas mujeres continuar sus actividades cotidianas, así como tener la capacidad de tomar decisiones. Hernández-Tezoquipa y colaboradores ${ }^{23}$ señalan que este tipo de acciones evita muchas veces que las mujeres caigan postradas y de este modo les permite continuar con su función primordial como "cuidadoras de los demás".
Por otra parte, la principal fuente de apoyo de las mujeres procede de la familia nuclear o las amigas, que juegan un papel central en las decisiones posteriores, así como en la elección de las estrategias de autoatención. En otros estudios se ha observado que cuanto más redes de apoyo existan, menos vulnerables son las mujeres y tienen mayores oportunidades de mejorar su salud emocional y por tanto su calidad de vida. ${ }^{24,25}$

Como se ha mencionado ya en los resultados, la pareja no se percibe como una importante fuente de apoyo. Para varias de las entrevistadas, la relación con la pareja fue, precisamente, la causa del malestar o un factor que complicó el ya existente. En este sentido, algunos autores plantean que la vida en pareja conlleva más ventajas, en relación con el apoyo social, para los hombres que para las mujeres. Esto tiene su explicación en los roles culturales establecidos para las mujeres, como ser "buena esposa" y "buena madre". Entre otros atributos, el papel de esposa implica atender las necesidades de la pareja y los hijos, antes que las suyas propias, lo cual es fuente constante de estrés, cansancio y preocupación. ${ }^{26,27}$ Por otro lado, Duncan ${ }^{28}$ señala que percibir apoyo por parte de la pareja es un aspecto fundamental que incide en la calidad de vida de las mujeres.

Por último, la información presentada confirma que la autoatención cumple funciones preventivas, diagnósticas y de tratamiento. Como plantean Menénde $z^{2} y$ Haro, ${ }^{1}$ lo central no es determinar lo erróneo o correcto de las explicaciones causales, diagnósticas, terapéuticas o de la manera de sobrellevar el malestar; sino comprender que cualquier afirmación que se realice desde los distintos ámbitos médicos será evaluada y filtrada por otros conocimientos que se encuentren disponibles.

\section{Agradecimientos}

Los autores agradecen y expresan su reconocimiento a las personas entrevistadas que compartieron sus experiencias para hacer posible este trabajo. El proyecto de investigación base de estos resultados recibió financiamiento del Instituto Nacional de Psiquiatría Ramón de la Fuente (INPRFM-DIES 4188) y del Consejo Nacional de Ciencia y Tecnología (SEP-2004-C01-46563).

\section{Referencias}

I. Haro J. Cuidados profanos: una dimensión ambigua en la atención de la salud. En: Perdiguero E, Comelles JM (ed.). Medicina y cultura. Estudios entre la antropología y la medicina. Barcelona: Bellaterra, 2000:10I-I62. 2. Menéndez E. Intencionalidad, experiencia y función: la articulación de los saberes médicos. Rev Antropol Soc 2005; 14:33-69.

3. Hoy B,Wagner L, Hall E. Self-care as a health resource of elders: an integrative review of the concept. Scand J Caring Sci 2007;21:456-466. 
4. Solsona M. La segunda transición demográfica desde la perspectiva de género. En: Solsona M (ed.). Desigualdades de género en los viejos y los nuevos hogares. Barcelona: Universidad Autónoma de Barcelona, 1996:17-46.

5. Osorio RM. La cultura médica materna en la atención a la salud materno-infantil. En: Ravelo P, Pérez-Gil S, Ramírez JC (coord.). Género y salud femenina. Experiencias de investigación en México. México: CIESASINNSZ-Universidad de Guadalajara, 1995:45-64.

6. Tindle H, Davis R, Phillips R, Eisenberg D. Trends in use of complementary and alternative medicine by US adults: 1997-2002. Altern Ther Health Med. 2005; I : 42-49.

7. Wu P, Fuller C, Liu X, Lee H, Fan B, Hoven C, et al. Use of complementary and alternative medicine among women with depression: results of a national survey. Psych Serv (Estados Unidos) 2007;58:349-356. 8. Berenzon S,Alanís S, Saavedra N. El uso de terapias alternativas y complementarias en población mexicana con trastornos depresivos y de ansiedad: resultados de una encuesta en la Ciudad de México. Salud Mental 2009;32:107-II5.

9. Solís L, Medina-Mora ME. La utilización de servicios de atención para la salud mental por mujeres mexicanas. Resultados de dos encuestas nacionales. Salud Mental 1994; 17:7-10.

10. Medina-Mora ME, Borges G, Lara C, Benjet C, Blanco J, Fleiz C, et al. Prevalencia de trastornos mentales y uso de servicios: resultado de la Encuesta Nacional de Epidemiología Psiquiátrica en México. Salud Mental 2003;26:I-16.

II. Mora-Ríos J, Ito-Sugiyama ME. Padecimientos emocionales, búsqueda de ayuda y expectativas de atención en una comunidad urbano-marginal. Salud Pública Mex 2005;47: 145-154.

12 Burin M. Poder, amor y sexualidad en la construcción de la subjetividad. Buenos Aires: Paidós, 1998.

13. Finkler K.Women in pain. Gender and morbidity in Mexico. Philadelphia: University of Pensylvania Press, 1994.

14. Glasser B, Strauss A. The discovery of grounded theory. Chicago: Aldine, 1967.

I5.Alonso L. Sujeto y discurso: el lugar de la entrevista abierta en las prácticas de la sociología cualitativa. En: Delgado JM, Gutiérrez J (ed.).
Métodos y técnicas de investigación en ciencias sociales. Madrid: Síntesis, 1995:225-240

16. Sierra F. Función y sentido de la entrevista cualitativa. En: Galindo J (coord.). Técnicas de investigación en sociedad, cultura y comunicación. México:Addison Wesley Longman, 1998:277-34I.

17. Kvale S. Interviews: an introduction to qualitative research interviewing. Londres: Sage Publications, 1996.

18. Salgado-de Snyder N, Díaz-Pérez MJ, González-Vázquez T. Modelo de integración de recursos para la atención de la salud mental en la población rural de México. Salud Pública Méx 2003;45:19.

19. Organización Mundial de la Salud. Perspectivas políticas sobre medicamentos de la OMS. Ginebra: Organización Mundial de la Salud, 2002.

20. Broadhead W, Gehlbach S, Degruy F, Kaplan B. Functional versus structural social support and health care utilization in a family medicine outpatient practice. Med Care 1989;27:709-723.

21.Argueta A, Cano L, Rodarte ME.Atlas de las plantas medicinales tradicionales mexicanas. México: Instituto Nacional Indigenista, 1994. 22. Hernández $\mathrm{H}$,Arenas ML,Valde R. El cuidado a la salud en el ámbito doméstico: interacción social y vida cotidiana. Rev Saúde Públ 200I;35:443-50.

23. Hernández-Tezoquipa I,Arenas-Monreal M,Valdez-Santiago R. No dejarse caer en cama. Las mujeres y los servicios de salud. Cad Saúde Públ 2005;21:1210-1216.

24. Hansson A, Hilleras P, Rosell Y. What kind of self care strategies do people report using and is there an association with well-being? Soc Indic Res 2005;73:133-139.

25. Sapag J, Kawachi I. Capital social y promoción de la salud en América Latina. Rev Saúde Públ 2007;41:139-149.

26. Mendias E, Clark M, Guevara E.Women's self-perception and self care practice: implications for health care delivery. Health Care Women Int 200I;22:299-3I2.

27. Matud P, Carballeira M, López M, Marrero R, Ibáñez I.Apoyo social y salud: un análisis de género. Salud Mental 2002;25:32-37.

28. Duncan K. How a supportive partner may increase relationship satisfaction. Br J Guid Counsell 2006;34:I 17-131. 